\title{
Journal für die \\ reine und angewandte Mathematik
}

\section{(Crelles Journal)}

\author{
GEGRÜNDET 1826 VON \\ August Leopold Crelle
}

FORTGEFÜHRT VON

Carl Wilhelm Borchardt · Karl Weierstrass · Leopold Kronecker Lazarus Fuchs · Kurt Hensel - Ludwig Schlesinger - Helmut Hasse Hans Rohrbach · Martin Kneser · Peter Roquette

\section{GEGENWÄRTIG HERAUSGEGEBEN VON}

Tobias H. Colding, Cambridge MA · Daniel Huybrechts, Bonn Jun-Muk Hwang, Seoul · Jakob Stix, Frankfurt am Main Rainer Weissauer, Heidelberg 
JOURNAL FÜR DIE REINE UND ANGEWANDTE MATHEMATIK

(CRELLES JOURNAL)

GEGRÜNDET 1826 VON

August Leopold Crelle

FORTGEFÜHRT VON

August Leopold Crelle (1826-1855)

Peter Roquette (1977-1998)

Carl Wilhelm Borchardt (1857-1881)

Samuel J. Patterson (1982-1994)

Karl Weierstrass (1881-1888)

Michael Schneider (1984-1995)

Leopold Kronecker (1881-1892)

Simon Donaldson (1986-2004)

Lazarus Fuchs (1892-1902)

Karl Rubin (1994-2001)

Kurt Hensel (1903-1936)

Joachim Cuntz (1994-2017)

Ludwig Schlesinger (1929-1933)

David Masser (1995-2004)

Helmut Hasse (1929-1980)

Gerhard Huisken (1995-2008)

Hans Rohrbach (1952-1977)

Eckart Viehweg (1996-2009)

Otto Forster (1977-1984)

Wulf-Dieter Geyer (1998-2001)

Martin Kneser (1977-1991)

Yuri I. Manin (2002-2008)

Willi Jäger (1977-1994)

Paul Vojta (2004-2011)

Horst Leptin (1977-1995)

Marc Levine (2009-2012)

GEGENWÄRTIG

HERAUSGEGEBEN VON

Tobias H. Colding

Daniel Huybrechts

Jun-Muk Hwang

Jakob Stix

Rainer Weissauer 
AUSGABEDATUM DES BANDES 783

Februar 2022

\section{CONTENTS}

T. Ikeda, H. Katsurada, An explicit formula for the Siegel series of a quadratic form over a non-archimedean local field. . . . . . . . . . . . . . . . . . . . . . . . . . . . . . . . . . . . . . . .

K.Y. Chan, Restriction for general linear groups: The local non-tempered Gan-Gross-Prasad conjecture

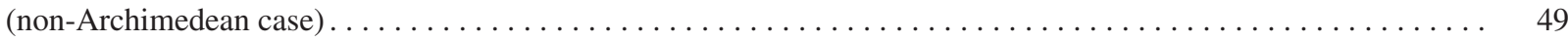

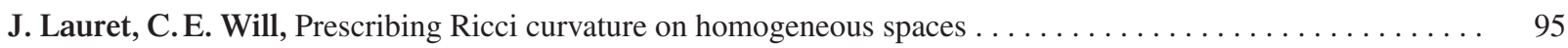

S. Gao, H. Li, X. Wang, Self-similar solutions to fully nonlinear curvature flows by high powers of curvature. . . 135

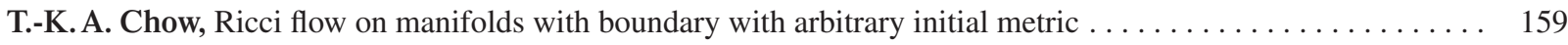

R. Cheng, R. van Dobben de Bruyn, Unbounded negativity on rational surfaces in positive characteristic. . . . . 217

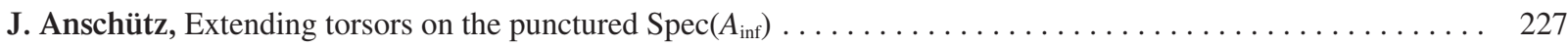

D. García-Lucas, L. Margolis, Á. del Río, Non-isomorphic 2-groups with isomorphic modular group algebras. . . 269

J. Ueki, Erratum to Profinite rigidity for twisted Alexander polynomials

(J. reine angew. Math. 771 (2021), 171-192) . . . . . . . . . . . . . . . . . . . . . . . . . . . . . . . . . 275 


\section{JOURNAL FÜR DIE REINE UND ANGEWANDTE MATHEMATIK (CRELLES JOURNAL)}

Crelle's Journal is devoted to the publication of original mathematical research papers. Contributions will be considered for publication in Crelle's Journal if they have not been published previously and are not under consideration for publication elsewhere.

All information regarding notes for contributors, subscriptions, Open access, back volumes and orders is available online at www.degruyter.com/crelle.

\section{EDITORS}

Tobias H. Colding

Department of Mathematics

Massachusetts Institute of Technology

77 Massachusetts Avenue

Cambridge, MA 02139-4307, USA

Email: journals@math.mit.edu

Daniel Huybrechts

Mathematisches Institut

Rheinische Friedrich-Wilhelms-Universität

Endenicher Allee 60

53115 Bonn, Germany

Email: huybrech@math.uni-bonn.de
Jun-Muk Hwang

Institute for Basic Science

Center for Complex Geometry

55 Expo-ro, Yuseoung-gu

Daejeon, Korea 34126

Email: jmhwang@ibs.re.kr

Jakob Stix

Institut für Mathematik

Johann Wolfgang Goethe-Universität

Robert-Mayer-Str. 6-8

60325 Frankfurt am Main, Germany

Email: stix.crelle@math.uni-frankfurt.de

\section{MANAGING EDITOR}

Rainer Weissauer

Mathematisches Institut

Ruprecht-Karls-Universität Heidelberg

Im Neuenheimer Feld 288

69120 Heidelberg, Germany

Email: weissauer.crelle@mathi.uni-heidelberg.de

ABSTRACTED/INDEXED IN Baidu Scholar · Cabells Journalytics · CNKI Scholar (China National Knowledge Infrastructure) · CNPIEC: cnpLINKer · Dimensions · EBSCO (relevant databases) · EBSCO Discovery Service · Genamics JournalSeek · Google Scholar · Japan Science and Technology Agency (JST) · J-Gate · Journal Citation Reports/Science Edition · JournalGuide · JournalTOCs · KESLI-NDSL (Korean National Discovery for Science Leaders) · Mathematical Reviews (MathSciNet) · Microsoft Academic $\cdot$ MyScienceWork $\cdot$ Naver Academic · Naviga (Softweco) · Primo Central (ExLibris) · ProQuest (relevant databases) · Publons · QOAM (Quality Open Access Market) · ReadCube · SCImago (SJR) · SCOPUS · Semantic Scholar · Sherpa/RoMEO · Summon (ProQuest) · TDNet · Ulrich's Periodicals Directory/ulrichsweb - WanFang Data - Web of Science: Current Contents/Physical, Chemical and Earth Sciences; Science Citation Index; Science Citation Index Expanded · WorldCat (OCLC) · Yewno Discover · zbMATH Open.

ISSN 0075-4102 · e-ISSN 1435-5345 · CODEN JRMAA8

RESPONSIBLE EDITORS see above

PUBLISHER Walter de Gruyter GmbH, Berlin/Boston, Genthiner Straße 13, 10785 Berlin, Germany

JOURNAL MANAGER Theresa Haney, De Gruyter, Genthiner Straße 13, 10785 Berlin, Germany. Tel.: +49 (0)30 260 05-375, Fax: +49 (0)30 260 05-250

Email: theresa.haney@ degruyter.com

RESPONSIBLE FOR ADVERTISEMENTS Markus Kügel, De Gruyter, Rosenheimer Str. 143, 81671 München, Germany.

Tel.: +498976 902-424

Email: anzeigen@degruyter.com

(C) 2022 Walter de Gruyter GmbH, Berlin/Boston

TYPESETTING WisSat Publishing + Consulting GmbH, Fürstenwalde

PRINTING Franz X. Stückle Druck und Verlag e.K., Ettenheim

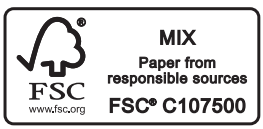

